\title{
Systemic Chemotherapy for Urothelial Cancer - How to Select Systemic Therapy in Bladder Cancer
}

\author{
Ioannis Dimitriadis and Aristotelis Bamias \\ Department of Clinical Therapeutics, National and Kapodistrian University of Athens, Athens, Greece
}

DOI: https://doi.org/10.17925/EOH.2017.13.02.134

$\mathrm{U}$ rothelial cancer (UC) has long been recognised as a chemosensitive disease, and systemic chemotherapy plays a crucial role in the management of localised and advanced disease. Unfortunately, there has been a paucity of progress during the last 15 years in this area. Neoadjuvant use of cisplatin-based combinations has shown survival benefit and together with radical cystectomy should constitute the cornerstone of early disease management. Most importantly, Galsky criteria concerning fitness for cisplatin provide a useful tool for clinicians, in order to select patients with substantial benefit from cisplatin-based chemotherapy. Adjuvant chemotherapy may be useful in selected cases, but its wide implementation has to be proved. Recently, the advent of modern immunotherapy seems to offer new effective choices for patients with advanced UC.

\section{Keywords}

Bladder cancer, urothelial cancer, immunotherapy, eligible for cisplatin, ineligible for cisplatin, transitional cell carcinoma

Disclosure: Ioannis Dimitriadis and Aristotelis Bamias have nothing to disclose in relation to this article.

Compliance with Ethics: This study involves a review of the literature and did not involve any studies with human or animal subjects performed by any of the authors.

Authorship: All named authors meet the International Committee of Medical Journal Editors (ICMJE) criteria for authorship of this manuscript, take responsibility for the integrity of the work as a whole, and have given final approval to the version to be published.

open Access: This article is published under the

Creative Commons Attribution Noncommercial License which permits any non-commercial use, distribution, adaptation and reproduction provided the original author(s) and source are given appropriate credit.

Received: 31 May 2017

Accepted: 15 August 2017

Citation: European Oncology \& Haematology, 2017;13(2):134-8

Corresponding Author: Ioannis Dimitriadis, Oncology-Hematology Unit, Department of Clinical Therapeutics, Alexandra Hospital, 80 Vas. Sofias, GR11528 Athens, Greece. E: dimitriadis.@yahoo.com

Support: No funding was received in

the publication of this article
Urothelial cancer (UC) is a common malignancy both in men and women. More than 400,000 patients are diagnosed every year and approximately 150,000 die from this disease, worldwide. ${ }^{1}$ At the same time, 5 -year survival rate has only been moderately increased during the last 30 years. ${ }^{2}$

UC may arise throughout the urinary tract (urinary bladder, ureter, renal pelvis, urethra) but more than $90 \%$ of them occur in the bladder. The major histological subtype is transitional-cell carcinoma (92\%), while pure squamous and adenocarcinomas are rare (2\%). ${ }^{3}$ Mixed variants are more common than pure non-transitional histologies. Tumour stage at diagnosis represents the most important prognostic indicator. Non-muscle invasive tumours (Tis, $\mathrm{Ta}$, $\mathrm{T} 1$ ) represent almost $70 \%$ of the newly diagnosed cases, and are associated with excellent prognosis (almost $90 \%$ 5 -year survival) compared to muscle-invasive ( $\geq T 2$ ) tumours (less than $65 \%$ survival). ${ }^{4}$

The chemosensitivity of UC has long been accepted. Taking into consideration that $20 \%$ of muscle-invasive tumours are associated with metastatic disease at diagnosis and 40-50\% of initially non-metastatic disease recurs after local therapy, it is obvious that the role of systemic chemotherapy is important in a sizable percentage of patients. Furthermore, the high probability of relapse after local therapy has introduced the concept of peri-operative chemotherapy as a means of reducing relapse rates. In this review, we discuss the current status of systemic chemotherapy in muscle-invasive UC.

\section{Chemotherapy for localised muscle-invasive urothelial cancer}

The first step in the management of muscle-invasive bladder cancer (MIBC) is transurethral resection of the bladder tumour (TURBT) aiming at maximal resection and accurate diagnosis of the disease. Smooth muscle must be included in the specimen. After TURBT, disease should be staged with at least computed tomography (CT) of the thorax, abdomen and pelvis. In case of no metastases, the optimal modality for local therapy should be considered and discussed with the patient. Traditionally, radical cystectomy accompanied by pelvic lymphadenectomy has been the standard of local treatment. ${ }^{5}$ Despite improvement in surgical techniques, however, locoregional or distant failure occurs in about $50 \%$ for T3 cases. ${ }^{6}$ In this setting, chemotherapy has been widely used to eliminate micrometastatic disease. ${ }^{7}$ In addition, a considerable proportion of patients cannot undergo cystectomy due to co-morbidities or noncompliance. ${ }^{8}$ Recent data, suggest that radiotherapy combined with chemotherapy can achieve comparable outcomes regarding local and distant control to radical cystectomy. ${ }^{9}$

\section{Neoadjuvant chemotherapy}

Preoperative (neoadjuvant) cisplatin-based chemotherapy is strongly recommended as standard treatment by both medical oncology (The National Comprehensive Cancer Network [NCCN], The European Society for Medical Oncology [ESMO]) and urological associations (The European 
Association of Urology [EAU]). - $^{-10}$ The first clinical trial investigating this approach was the BA06 30894 trial which administered three cycles of cisplatin, methotrexate and vinblastine (CMV) prior to definitive radical cyctectomy or radiotherapy versus definitive local therapy alone. Long term follow-up revealed $16 \%$ decrease in the risk of death and almost a $6 \%$ absolute increase in 10-year survival rate. ${ }^{11}$

The second well-designed randomised study (SWOG 8710/INT-0080) compared treatment with the MVAC regimen (methotrexate-vinblastinedoxorubicin-cisplatin) followed by radical cystectomy versus radical cystectomy alone. A clinically meaningful improvement in overall survival (OS) was revealed on final analysis (77 versus 46 months; $p=0.06$ ). Importantly, response to MVAC was an important prognostic factor: no evidence of residual disease (PT0) at cystectomy after neoadjuvant chemotherapy, predicted an OS of $85 \%$ at 5 years. ${ }^{12}$ Although MVAC proved its efficacy as preoperative treatment, its toxicity (44\% Grade 4 neutropenia) limits its use. Consequently, newstrategieswere implemented in order to reduce toxicity and lead to shorter diagnosis-to-cystectomy times. Dose-dense MVAC, given every 15 days with granulocyte-colony stimulating factor (GCSF) support was tested versus conventional MVAC in the metastatic setting, leading to a statistically significant improvement in median progression-free survival (PFS: 9.1 versus 8.2 months; $p=0.037){ }^{13}$ while long-term follow up suggested improvement in OS too..$^{14}$ These data were extrapolated in the neoadjuvant setting and were consistent with the findings of two single-arm, phase II trials. ${ }^{14,15}$

Another combination gaining use in the preoperative setting is the gemcitabine-cisplatin (GC) regimen. GC has shown similar efficacy and lower toxicity than MVAC in the metastatic setting. ${ }^{16}$ Real-world data by Galsky et al. revealed that GC is more commonly used as neoadjuvant chemotherapy leading to similar pathalogic complete response $(\mathrm{pCR})$ rates and survival outcomes. ${ }^{17}$ These data were also confirmed in retrospective data from 19 institutions internationally. ${ }^{18}$ The GC combination has recently been investigated in its dose-dense form, and given the fact that it has produced similar results in phase $\|$ trials, it is anticipated to gain more acceptance in clinical practice. ${ }^{19}$

Despite the high level of evidence supporting its use, neoadjuvant chemotherapy is underutilised. Several reasons have been suggested to explain this deviation from existing guidelines. The theoretical detrimental effect of delaying definitive treatment during neoadjuvant chemotherapy accounts for the reluctance of urologists to refer patients for this modality. In addition, only $50 \%$ of candidates for neoadjuvant chemotherapy are eligible for cisplatin-based combination chemotherapy, ${ }^{20}$ which is the only treatment that showed a benefit from this approach. Recent advances in molecular classification of MIBC have produced interesting results in identifying patients who are more likely to benefit from neoadjuvant chemotherapy and this way can prevent its utilisation in patients who are less likely to respond or these who are going to have excellent progonosis with cystectomy alone. ${ }^{21}$ Fortunately, according to National Cancer Institute database, there has been a recent increase in the percentage of patients on neoadjuvant approach, from $7.6 \%$ in 2006 to $20.9 \%$ in $2010 .{ }^{22}$ The importance of multidisciplinary approach and careful selection of patients for neoadjuvant chemotherapy are critical factors contributing to this progress.

\section{Adjuvant chemotherapy}

Reduction of recurrence rate and thus, prolongation of survival, is the rationale behind the use of adjuvant chemotherapy. This is more relevant when disease is not confined to the bladder (pT3-pT4, $\mathrm{N}+$ disease). The 5 -year survival rate is $30 \%$ for $\mathrm{N}+$ disease and almost $50 \%$ for $\mathrm{T} 3$
Figure 1: Proposed algorithm for eligible for cystectomy muscle-invasive bladder cancer

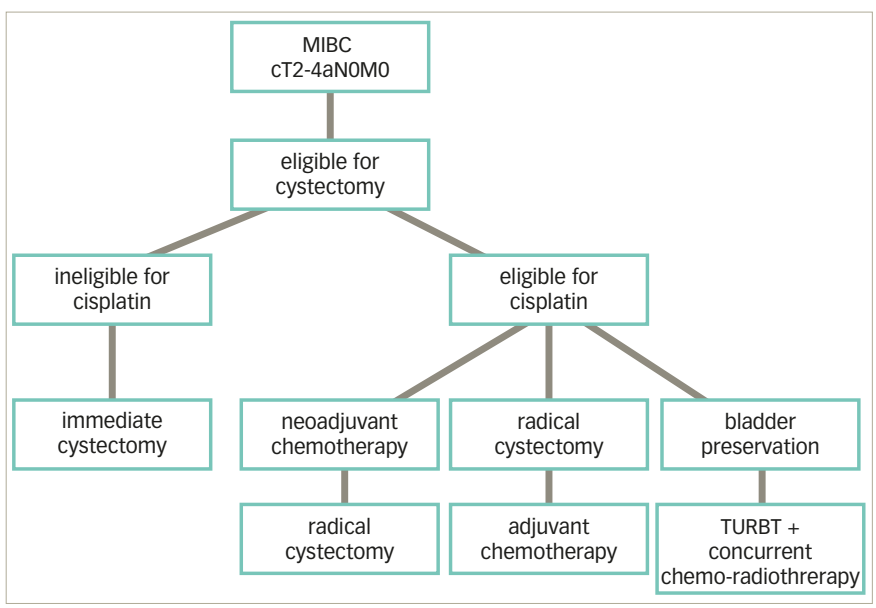

MIBC = muscle-invasive bladder cancer; $T$ TURBT = transurethral resection of the bladder tumour. Modified with permission from Zagouri et al..$^{59}$

disease. ${ }^{23}$ It should be stressed that all available evidence in this context is associated with cisplatin-based chemotherapy. We should stress that around $40 \%$ of candidates do not have adequate renal function to undergo adjuvant chemotherapy. ${ }^{24,25}$

Few randomised trials have evaluated the role of adjuvant chemotherapy for UC. Most of them were stopped early due to poor accrual. Review of these trials revealed a number of methodological flaws: low accrual with insufficient sample size, ineffective chemotherapy regimens, inadequate statistical methodology. ${ }^{26-32} \mathrm{~A}$ meta-analysis of six early randomised trials, including 491 patients, published in 2005, revealed a global reduction of the risk of death of $25 \%$ (hazard ratio $[\mathrm{HR}]=0.75$, $95 \%$ confidence interval [Cl] $0.60-0.96, p=0.019$ ) with an absolute gain in survival of $9 \%$ at 3 years. ${ }^{33}$

The largest randomised trial on adjuvant treatment was published by European Organisation for Research and Treatment of Cancer (EORTC; trial 30994). ${ }^{34}$ It was also stopped early due to slow accrual after randomising 284 patients instead of the pre-planned number of 660 . It failed to meet its primary endpoint concerning OS benefit (adjusted HR $0.78,95 \% \mathrm{Cl} 0.56-1.08, \mathrm{p}=0.13$ ) but it showed a significant increase in 5-years PFS for adjuvant chemotherapy arm (HR 0.54, 95\% Cl 0.4-0.73, $p<0.0001)$.

To conclude, adjuvant chemotherapy on UC remains a topic of debate. In spite of obvious advantages (administration without delay of surgical procedures, accurate staging based on cystectomy specimen) it is not supported by the level of evidence associated with neoadjuvant chemotherapy, which remains the standard perioperative therapy in bladder cancer. Nevertheless, EAU, ESMO and NCCN guidelines suggest the use adjuvant chemotherapy in high risk patients after cystectomy (extra-vesical or node-positive disease) who did not receive neoadjuvant chemotherapy (Figure 1). ${ }^{8-10}$

\section{Bladder-preservation approach - non-cystectomy candidate}

A sizable proportion of patients with MIBC are unfit for or unwilling to undergo cystectomy. This has fuelled research on bladder-preservation strategies as an alternative to radical cystectomy. Bladder-preserving strategies ideally involve a tri-modality approach: maximal TURB-T followed by combined radio-chemotherapy (Figure 2). 
Figure 2: Proposed algorithm for those ineligible for cystectomy muscle-invasive bladder cancer

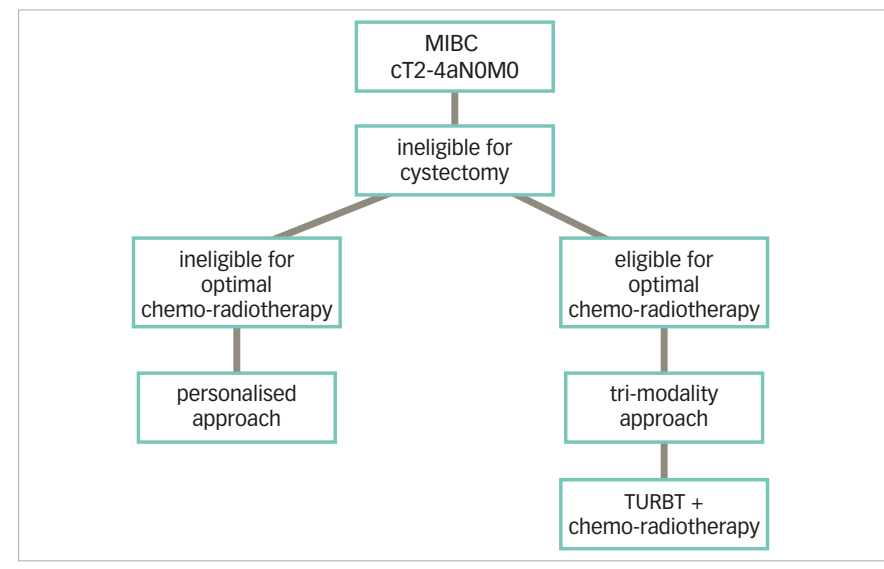

MIBC = muscle-invasive bladder cancer; $T$ TURBT $=$ transurethral resection of the bladder tumour. Modified with permission from Zagouri et al. ${ }^{59}$

This importance of the addition of chemotherapy to radiotherapy was recently shown by James et al.: the addition of chemotherapy ith 5-fluorouracil (5FU) and Mitomycin-C to radical radiation, increased locoregional control, which was considered a surrogate for OS. ${ }^{35}$ Although cisplatin is the most studied drug in the concurrent chemotherapy setting, this is a useful approach, specifically in the cisplatin-ineligible population.

The role of neoadjuvant chemotherapy in the context of radiochemotherapy remains unclear. Subanalyses of the previously mentioned trial suggested a benefit by the administration of chemotherapy prior to the combined modality but the true value of this strategy can only be defined in randomised trials.

\section{Chemotherapy for advanced urothelial cancer}

Chemotherapy represents the mainstay of management of advanced (pT4b, or $\mathrm{N}+$ ) or metastatic disease (M1). Nevertheless, multidisciplinary approach and local control, remain crucial factors to obtain optimal results especially in patients with lymph node disease only. ${ }^{36}$

Cisplatin-based combination chemotherapy represents the treatment of choice in advanced disease. The combination of methotrexate, vinblastine, doxorubicin and cisplatin (MVAC) produced superior response rates and survival than single agent cisplatin (12.5 versus 8.5 months); although, at the cost of increased toxicity. ${ }^{37}$ The prognosis of patients following cisplatin-based chemotherapy has been defined by the MSKCC algorithm, which combines Karnofsky performance status (KPS) and the presence of visceral metastases. ${ }^{38-39}$

Efforts to improve upon the efficacy of MVAC have failed, but more tolerable regimes have been developed. The EORTC 30924 trial investigated the dose dense (DD) (or accelerated) version of MVAC in an effort to control haematological toxicity. ${ }^{40}$ DD-MVAC showed a significant increase in complete response (CR) (21 versus 9\% in the standard MVAC standard arm, $\mathrm{p}=0.009$ ) and in PFS ( 9.1 months, compared with 8.2 months with MVAC, $p=0.037$ ). After 2 years, the probability to be free from progression was more than doubled for patients receiving DDMVAC (24.7 versus $11.6 \%, p=0.037$ ). Although survival seems to favour the DD-MVAC arm, the difference was not statistically significant $(p=0.122)$. DD-MVAC permitted delivery of twice the dose of cisplatin and adriamycin compared to MVAC with less haematologic toxicity due to the use of GCSF. ${ }^{40}$
A phase III randomised trial of 405 patients was conducted to compare GC with MVAC. ${ }^{40,41}$ Response rates (49\% versus $46 \%$ ), time to progression (median: 7.4 versus 7.4 months) and OS rates (median OS 13.8 versus 14.8 months; $p=0.75$ ) were similar in both arms. More importantly, GC was better tolerated than MVAC, with $63 \%$ of cycles administered with no dose modifications compared with $37 \%$ dose modifications in the MVAC arm. Patients on the GC arm experienced less grade 3 or 4 neutropenia (71\% versus $82 \%$ ), neutropenic fever ( $2 \%$ versus $14 \%$ ), and neutropenic sepsis (1\% versus $12 \%)$; and grade 3 or 4 mucositis was significantly less common on the GC arm (1\% versus $22 \%$ ), as was the toxic death rate (1\% versus $3 \%){ }^{40}$ The equivalent efficacy and better tolerability profile of the $\mathrm{GC}$ regimen has led to its adoption as the preferred, standard firstline treatment for patients with locally advanced or metastatic bladder cancer in the community.

Dose-dense GC with GCSF support was compared to DD-MVAC in a Greek trial. ${ }^{42}$ Efficacy (median OS, median PFS) were similar between the two groups (DD-MVAC versus DD-GC; median OS: 19 versus 18 months; $p=0.98$, median PFS: 8.5 versus 7.8 months; $p=0.36$ ). Toxicity marginally favoured DD-GC (grade $\geq 350 \%$ DD-MVAC versus $44 \%$ DDGC) and significantly more patients received at least six cycles of DD-GC compared with DD-MVAC (85 versus 63\%; $p=0.011$ ). Thus, both DD-GC and DD-MVAC remain viable options in the cisplatin-eligible population.

Platinum-containing triplets or non-platinum containing regimens have been studied in a series of trials. They have produced interesting results but the toxicity profile of triplets and the well-established results of platinum-containing doublets have turned them not applicable in everyday practice. Specifically, PGC regimen (paclitaxelgemcitabine-cisplatin) was studied in the EORTC 30987 trial, ${ }^{43}$ but failed to produce significant benefit in OS (15.8 months with PGC versus 12.7 months with $\mathrm{GC}$; HR $0.85,95 \% \mathrm{Cl} 0.72-1.02, \mathrm{p}=0.075$ ) or PFS (8.3 months with PCG, 7.6 months with GC; HR 0.87, 95\% Cl $0.74-$ $1.03, p=0.113$ ). Only overall response rate (ORR) improved (55.5 versus $43.6 \%$ for $P C G$ versus $G C$, respectively, $\mathrm{p}=0.0031$ ) to the cost of more febrile neutropenia in PGC.

Cisplatin ineligible patients comprise about $50 \%$ of the total population of advanced UC.20 Carboplatin-based chemotherapy has been proposed as the standard in this population. The EORTC 30986 phase II/III trial compared gemcitabine-carboplatin (GCa) combination with methotrexate-carboplatin and cinblastine (M-CAVI), obtaining similar results in terms of OS (8.1 versus 9.3 months for patients treated with $\mathrm{M}$-CAVI and GCa, respectively, $\mathrm{p}=0.64$ ) and PFS (4.2 months for M-CAVI arm and 5.8 months for $\mathrm{GCa}, \mathrm{p}=0.75$ ) but a significantly better toxicity profile in patients treated with GCa. ${ }^{44}$ De Santis et al. investigated a non-carboplatin regimen, vinlunine-gemcitabine, versus vinfluninecarboplatin in the JASINT1 trial. ${ }^{45}$ Both doublets offered similar PFS (5.9 versus 6.1 months respectively) and OS (14 versus 12.8 months) results. It should be noted that only performance status (PS) 0 and 1 patients were eligible for enrolment, which makes this population more favourable than a typical cisplatin-ineligible cohort. The value of carboplatin in the unfit population is clearly shown in the data from the RISC database where carboplatin treated patients had a longer survival compared to the untreated population (9.5 versus 6.8 months). ${ }^{46}$ Nevertheless, prognosis remains poor and more effective therapies are urgently needed for these patients.

Immunotherapy is probably the most promising new comer in management of metastatic UC. Its role is more evident in the unfit for cisplatin population. Results from IMvigor210 trial cohort 1, regarding the 
use of atezolizumab (anti programmed death-ligand 1 [PD-L1] antibody) as first-line therapy in cisplatin-ineligible locally advanced/metastatic UC, show that responses occur in all subgroups, irrespective of PD-L1 status (22\% IC2/3, 19\% all). ${ }^{47}$ Pembrolizumab has also produced meaningful and durable responses in the same population in the KEYNOTE-052 trial. ${ }^{48}$ With a median follow-up time of 7.8 months, the ORR was $28.6 \%$ $(95 \% \mathrm{Cl} 24,34)$ and the median response duration was not reached (range $+1.4,+17.8$ months). These results have led to FDA approval of atezolizumab and pembrolizumab as first-line treatment in the cisplatinineligible population. More effort is ongoing concerning incorporating immunotherapy in first-line setting, and this is going to be answered by the IMvigor130 trial which has been recently modified to compare atezolizumab monotherapy versus its combination with chemotherapy in both cisplatin-eligible and -ineligible groups. ${ }^{49}$

Most patients with advanced UC will relapse after first-line treatment. Karnofsky Performance Score (KPS), liver metastatic disease, and haemoglobin level are significant prognostic factors in this setting. ${ }^{50}$ The optimal treatment for these patients remains undefined. A meta-analysis by Raggi et al. proved that combinations did not provide any significant improvement in OS, but only a statistically significant benefit in response rate. ${ }^{51}$ Therefore, they are only suggested when disease control matters in case of severe symptoms.

ESMO guidelines suggest choosing platinum-rechallenge based on the first-line PFS (Figure 3); ${ }^{10}$ although, this practice is not supported by a high level of evidence. Vinflunine, a novel vinca alkaloid, is only approved in Europe for second-line treatment of UC, based on the results of a phase III trial, where a significant survival benefit over best supportive care was shown only in the patients treated according to study protocol. ${ }^{22}$ Lately, angiogenesis inhibition using the combination of a vascular endothelial growth factor receptor (VEGFR)-2 inhibitor, ramucirumab with docetaxel has shown statistically significant prolongation of PFS in a phase III trial; more data on OS are anticipated before regulatory approval. ${ }^{53}$

\section{Immunotherapy in second-line treatment of urothelial cancer}

Lately, the landscape of second-line treatment seems to be changing with the evolving role of immunotherapy. In a phase III trial, pembrolizumab produced significantly longer OS compared to singleagent chemotherapy. In this trial (KEYNOTE-045) pembrolizumab was compared to standard second-line chemotherapy (taxane-based, vinflunine). ${ }^{54}$ Significant prolongation of OS was recorded in both unselected (10.3 versus 7.4 months) and PD-L1 selected population (8 versus 5.2 months). This is the first time, a novel agent demonstrates activity in prolonging OS on this setting compared to chemotherapy. In addition, the non-randomised IMvigor210 study showed promising results in a similar population. ${ }^{55}$ Stratification of patients according to PD-L1 status was associated with higher benefit, but considerable efficacy was also observed among PD-L1 non-expressors. Nivolumab, in the CheckMate275 trial, has also proven its efficacy in the same setting, producing high response rates irrespective of the PD-L1 expression. ${ }^{56}$
Figure 3: Proposed algorithm for the management of metastatic urothelial cancer based on the ESMO Guidelines ${ }^{10}$

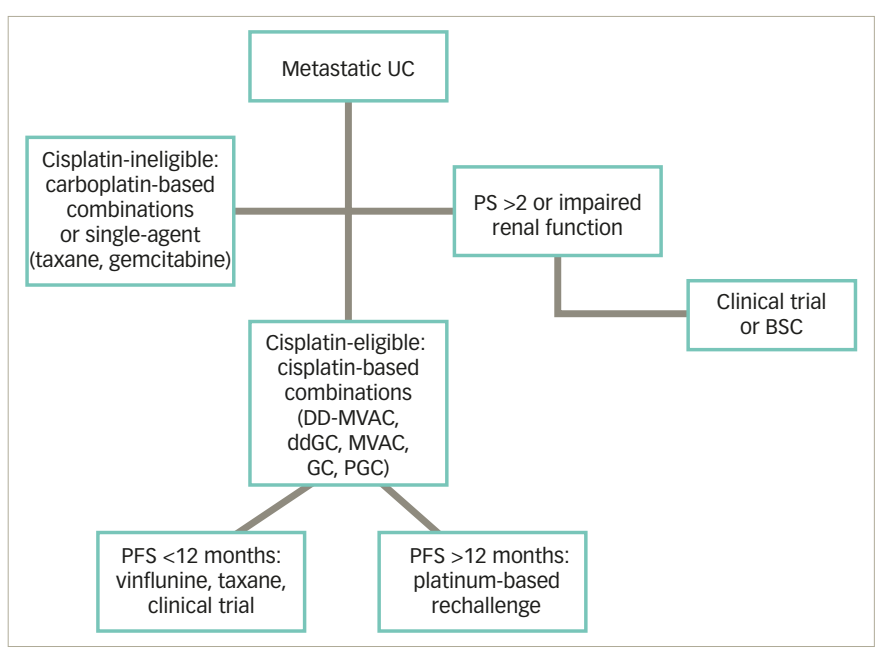

$B S C=$ best supportive care; $D D-G C=$ dose dense gemcitabine-cisplatin; DD-MVAC = dose dense methotrexate-vinblastine-doxorubicin-cisplatin; $G C=$ gemcitabinecisplatin; $M V A C=$ methotrexate-vinblastine-doxorubicin-cisplatin; $P F S=$ progression free survival; $P G C=$ paclitaxel-gemcitabine-cisplatin; $P S=$ performance status; $U C=$ urothelial cancer. Modified from Bellmunt et al. ${ }^{10}$

Therefore, other markers, such as mutational load or molecular subtype are currently studied as potential means of selection of patients likely to benefit from these therapies. ${ }^{57}$

\section{Conclusion}

UC has long been recognised as a chemosensitive disease, and systemic chemotherapy plays a crucial role in the management of localised and advanced disease. Unfortunately, there has been a paucity of progress during the last 15 years, in this area. Recently, the advent of modern immunotherapy seems to offer new effective choices for patients with advanced UC.

Neoadjuvant use of cisplatin-based combinations has shown survival benefit, and together with radical cystectomy should constitute the cornerstone of early disease management. ${ }^{8-10}$ Most importantly, Galsky criteria concerning fitness for cisplatin provide a useful tool for clinicians, in order to select patients with substantial benefit from cisplatin-based chemotherapy.

The discovery of predictive biomarkers to determine the most effective therapeutic strategy for a given patient is critical. This holds true for cytotoxic chemotherapy as well as for immunotherapy. Genetic profiling seems to be worth studying in this context. On the other hand, PD-L1 expression on UC cells has been correlated with a worse prognosis, but unfortunately, PD-L1 expression on tumour and immune cells has proven to be unreliable as the sole determinants of response to PD-1 blockade. ${ }^{58}$ Technical issues, tumour heterogeneity and the dynamic nature of its expression, may represent some of the reasons for this limitation. More insightful knowledge of immune system principles could improve our efforts to explore new biomarkers in advanced UC. $\square$
1. Ferlay J, Soerjomataram I, Ervik M et al., GLOBOCAN 2012 v1.1, Cancer Incidence and Mortality Worldwide: IARC CancerBase No.11. Lyon, France: International Agency for Research on Cancer; 2014. Available at: http://globocan.iarc.fr (accessed 5 May 2017).

2. Siegel R, Naishadham D, Jemal A. et al., Cancer statistics, 2013, CA Cancer I Clin, 2013;63:11-30.

3. Eble JN, Sauter G, Epstein JI, Sesterhenn IA (Eds), World health organization classification of tumors. Pathology and genetics of tumours of the urinary system and male genital organs. Lyon: IARC press; 2004.

4. American Cancer Society. Bladder Cancer; Early Detection,
Diagnosis, and Staging Topics. Available at: www.cancer.org/ cancer/bladder-cancer/detection-diagnosis-staging.html (accessed 5 May 2017).

5. Gakis G, Efstathiou J, Lerner SP et al., ICUD-EAU International Consultation on Bladder Cancer 2012: radical cystectomy and bladder preservation for muscle-invasive urothelial carcinoma of the bladder, Eur Urol, 2013;63:45-57.

6. Hautmann RE, Gschwend JE, de Petriconi RC et al Cystectomy for transitional cell carcinoma of the bladder: results of a surgery only series in the neobladder era, J Urol, 2006;176:486 92.

7. Galsky MD, Hahn NM, Rosenberg J, et al., A consensus definition of patients with metastatic urothelial carcinoma who are unfit for cisplatin-based chemotherapy, Lancet Oncol, 2011;12:211-4.

8. Clark PE, Spiess PJ, Agarwal N et al., NCCN Clinical Practice Guidelines in Oncology: Bladder Cancer, 2016. Available at: https://www.nccn.org/professionals/physician_gls/f_guidelines. asp\#\#bladder (accessed 5 May 2017).

9. Witjes J, Comperat $\mathrm{E}$, Cowan $\mathrm{N}$ et al., EAU guidelines on muscle-invasive and metastatic bladder cancer. European Association of Urology, 2015. Available at: https://uroweb.org/ individual-guidelines/oncology-guidelines/ (accessed 5 May 2017). 
10. Bellmunt A, Orsola A, Leow JJ et al., Bladder cancer: ESMO Practice Guidelines for diagnosis, treatment and follow-up, Ann Oncol, 2014;25 Suppl 3:iii40-8.

11. Neoadjuvant cisplatin, methotrexate and vinblastine chemotherapy for muscle-invasive bladder cancer: randomised controlled trial. International collaboration of trialists, Lancet, 1999;354:533-40.

12. Grossman HB, Natale RB, Tangen CM et al., Neoadjuvan chemotherapy plus cystectomy compared with cystectomy alone for locally advanced bladder cancer, N Eng/ J Med, 2003;349:859-66.

13. Sternberg C, De Mulder P, Schornagel J, et al., Seven year update of an EORTC Phase III trial of high-dose intensity M-VAC chemotherapy and G-CSF versus classic M-VAC in advanced urothelial tract tumours, Eur J Cancer, 2006;42:50-5.

14. Plimack ER, Hoffman-Censits JH, Viterbo R, et al., Accelerated methotrexate, vinblastine, doxorubicin, and cisplatin is safe, effective, and efficient neoadjuvant treatment for muscleinvasive bladder cancer: results of a multicenter Phase II study with molecular correlates of response and toxicity, I Clin Oncol with molecular cor

15. Choueiri TK, Jacobus S, Bellmunt J, et al., Neoadjuvant dosedense methotrexate, vinblastine, doxorubicin, and cisplatin with pegfilgrastim support in muscle-invasive urothelial cancer pathologic, radiologic, and biomarker correlates, J Clin Oncol, 2014;32:1889-94.

16. Von Der Maase H, Sengelov L, Roberts JT, et al., Long-term survival results of a randomized trial comparing gemcitabine plus cisplatin, with methotrexate, vinblastine, doxorubicin plus cisplatin in patients with bladder cancer, J Clin Oncol, 2005;23:4602-8

17. Galsky MD, Pal SK, Chowdhury S, et al., Comparative effectiveness of gemcitabine plus cisplatin versus methotrexate, vinblastine, doxorubicin, plus cisplatin as neoadjuvant therapy for muscle-invasive bladder cancer,

18. Zargar H, Espiritu PN, Fairey AS, et al., Multicenter assessment of neoadjuvant chemotherapy for muscle-invasive bladder cancer, Eur Urol, 2015;67:241-9.

19. Plimack E, Hoffman-Censits J, Kutikov A, et al., Neoadjuvant dose-dense gemcitabine and cisplatin (DDGC) in patients (pts) with muscle-invasive bladder cancer (MIBC): Final results of a multicenter phase II study, J Clin Oncol, 2014;32:5(suppl; abstr 4513).

20. Bamias A, Tzannis K, Liontos M, et al., Adherence to cisplatinbased regimens prescription in "fit" patients fulfilling platinum eligibility criteria. Impact on outcomes: a retrospective international study of invasive/advanced cancer of the urothelium (RISC) analysis, Ann Oncol, 2016;27:799.

21. Seiler R, Winters B, Douglas J, et al., Muscle-invasive bladder cancer: Molecular subtypes and response to neoadjuvant chemotherapy I Clin Oncol, 2017:35:Abstract 281

22. Zaid HB, Patel SG, Stimson CJ, et al., Trends in the utilization of neoadjuvant chemotherapy in muscle-invasive bladder cancer: results from the National Cancer Database, Urology, 2014;83:75-80

23. Von Rundstedt F-C, Mata DA, Kryvenko ON, et al., Utility of Clinical Risk Stratification in the Selection of Muscle-Invasive Bladder Cancer Patients for Neoadjuvant Chemotherapy: A Retrospective Cohort Study, Bladder Cancer (Amsterdam, Netherlands), 2016;3:35-44.

24. Bassi P, Ferrante GD, Piazza N, et al., Prognostic factors of outcome after radical cystectomy for bladder cancer: a retrospective study of a homogeneous patient cohort, J Urol, 1999:161:1494-7.

25. Donat SM, Shabsigh A Savage C, et al Potential impact of postoperative early complications on the timing of adjuvant chemotherapy in patients undergoing radical cystectomy: a high-volume tertiary cancer center experience, Eur Urol 2009;55:177-85

26. Skinner DG, Daniels JR, Russell CA, et al., The role of adjuvant chemotherapy following cystectomy for invasive bladdercancer: a prospective comparative trial, $J$ Urol, 1991;145:459-67.
27. Stockle M, Meyenburg W, Wellek S et al., Advanced bladder cancer (stages pT3b, pT4a, pN1 nad pN2): improved survival after radical cystectomy and 3 adjuvant cycles of chemotherapy results of a controlled prospective study, J Urol, 1992:148:302-7.

28. Stockle M, Meyenburg W, Wellek S, Adjuvant poylchemotherapy of nonorgan-confined bladder cancer after radical cystectomy revisited: Iong term results of a controlled prospective study and further clinical experience, J Urol, 1995; 153:47-52.

29. Freiha F, Reese J, Torti FM, A randomized trial of radica cystectomy versus radical cystectomy plus cisplatin, vinblastine and methotrexate chemotherapy for muscle-invasive bladder cancer, J Urol, 1996;155:495-9.

30. Studer UE, Bacchi M, Biedermann C, et al., Adjuvant cisplatin chemotherapy following cystectomy for bladder cancer: results of a prospective randomized trial, $J$ Urol, 1994;152:81-4.

31. Bono AV, Benvenuti C, Reali L, et al., Adjuvant chemotherapy in advanced bladder cancer. Italian Uro-Oncologic Cooperative Group, Prog Clin Biol Res, 1989;303:533-40.

32. Sylvester $R$, Sternberg $C$, The role of adjuvant combination chemotherapy after cystectomy in locally advanced bladder cancer:What we do not know and why, Ann Oncol 2000;11:851-6.

33. Advanced Bladder Cancer (ABC) Meta-analysis Collaboration. Adjuvant chemotherapy in invasive bladder cancer: a systematic review and meta-analysis of individual patient data Advanced Bladder Cancer DABC] meta-analysis collaboration Eur Urol, 2005;48:189-99. discussion 199-201.

34. Sternberg CN, Skoneczna I, Kerst JM et al., Immediate versus deferred chemotherapy after radical cystectomy in patients with pT3-pT4 or N+MO urothelial carcinoma of the bladder EORTC 30994]: an intergroup, open-label, randomised phase 3 trial, Lancet Oncol, 2015:16:76-86.

35. James ND, Hussain SA, Hall E, et al., Radiotherapy with or without chemotherapy in muscle-invasive bladder cancer N Engl J Med, 2012;366:1477-88.

36. Galsky M, Stensland A, Sfakianos J, et al., Comparative Effectiveness of Treatment Strategies for Bladder Cancer With Clinical Evidence of Regional Lymph Node Involvement, I Clin Onc, 2016;34:2627-35

37. Loehrer P, Einhorn LH, Elson PJ, et al., A randomized comparison of cisplatin alone or in combination with methotrexate, vinblastine, and doxorubicin in patients with metastatic urothelial carcinoma: a Cooperative Group Study, J Clin Oncol, 1992;10:1066-73.

38. Galsky MD, Moshier E, Krege S, et al., Nomogram for predicting survival in patients with unresectable and/or metastatic urothelial cancer who are treated with cisplatin-based chemotherapy, Cancer, 2013;119:3012-9.

39. Bajorin DF, Dodd PM, Mazumdar M, et al., Long-term survival in metastatic transitional cell carcinoma and prognostic factors predicting outcome of therapy I Clin Oncol, 1999:17:3173-81.

40. Sternberg $\mathrm{CN}$, de Mulder PH, Schornagel JH, et al., Randomized phase III trial of high-dose-intensity methotrexate, vinblastine, doxorubicin, and cisplatin (MVAC) chemotherapy and recombinant human granulocyte colony-stimulating factor versus classic MVAC in advanced urothelial tract tumors: European Organization for Research and Treatment of Cancer Protocol no. 30924, J Clin Oncol, 2001;19:2638-46.

41. von der Maase $\mathrm{H}$, Sengelov L, Roberts JT, et al., Long-term survival results of a randomized trial comparing gemcitabine plus cisplatin, with methotrexate, vinblastine, doxorubicin, plus cisplatin in patients with bladder cancer, $J$ Clin Oncol 2005:23:4602-8

42. Bamias A, Dafni U Karadimou A et al. Prospective, open-label, randomized, Phase III study of two dose-dense regimens MVAC versus gemcitabine/cisplatin in patients with inoperable metastatic or relapsed urothelial cancer: a Hellenic Cooperative Oncology Group study (HE 16/03), Ann Oncol, 2013;24:1011-1017.

43. Bellmunt J, von der Maase $\mathrm{H}$, Mead GM, et al., Randomized phase III study comparing paclitaxel/cisplatin/gemcitabine and gemcitabine/cisplatin in patients with locally advanced or metastatic urothelial cancer without prior systemic therapy:
EORTC Intergroup Study 30987, J Clin Oncol, 2012:30:1107-13. 4. De Santis M. Bellmunt J, Mead G, et al., Randomized Phase II III trial assessing gemcitabine / carboplatin and methotrexate carboplatin / vinblastine in patients with advanced urothelial cancer who are unfit for cisplatin-based chemotherapy: EORTC study 30986, I Clin Oncol, 2012;30:191-9.

45. De Santis M, Wiechno PJ, Bellmunt J, et al., Vinfluninegemcitabine versus vinflunine-carboplatin as first-line chemotherapy in cisplatin-unfit patients with advanced urothelial carcinoma: results of an international randomized phase II trial (JASINT1), Ann Oncol, 2016;27:449-54.

46. Necchi A, Sonpavde G, Lo Vullo S, et al., Nomogram-based prediction of overall survival (OS) of patients (pts) with metastatic urothelial carcinoma (UC) receiving first-line platinum-based chemotherapy: retrospective internationa study of invasive/advanced cancer of the urothelium (RISC), Ann Oncol, 2016:27:266-95.

47. Balar A, Galsky M, Rosenberg J, et al., Atezolizumab as first-line treatment in cisplatin-ineligible patients with locally advanced and metastatic urothelial carcinoma: a single-arm, multicentre, phase 2 trial, Lancet, 2017:389:67-76.

48. Balar A, Castellano D, O'Donnell P, et al., Pembrolizumab as first-line therapy in cisplatin-ineligible advanced urothelial cancer: Results from the total KEYNOTE-052 study population, J Clin Oncol, 2017;35, no. 6_suppl, abstract 284.

49. Study of Atezolizumab as Monotherapy and in Combination With Platinum-Based Chemotherapy in Participants With Untreated Locally Advanced or Metastatic Urothelial Carcinoma (IMvigor130): ClinicalTrials.gov Identifier: NCT02807636. Available at: https://clinicaltrials.gov/ct2/show/NCT02807636 (accessed 7 September 2017)

50. Bellmunt J, Choueiri TK, Fougeray R, et al., Prognostic factors in patients with advanced transitional cell carcinoma of the urothelial tract experiencing treatment failure with platinumcontaining regimens, I Clin Oncol, 2010:28:1850-5.

51. Raggi D, Miceli R, Sonpavde G, et al., Second-line singleagent versus doublet chemotherapy as salvage therapy for metastatic urothelial cancer: a systematic review and metaanalysis, Ann Oncol, 2016;27:49-61.

52. Bellmunt J, Théodore C, Demkov T, et al., Phase III trial of vinflunine plus best supportive care compared with best supportive care alone after a platinum containing regimen in patients with advanced transitional cell carcinoma of the urothelial tract, J Clin Oncol, 2009;27:4454-61.

53. Petrylak DP, de Wit R, Chi KN et al., Ramucirumab plus docetaxel versus placebo plus docetaxel in patients with locally advanced or metastatic urothelial carcinoma after platinum-based therapy (RANGE): a randomised, double-blind phase 3 trial, Lancet,2017; pii:S0140-6736(17)32365-6. [Epub ahead of print].

54. Bellmunt J, de Wit R, Vaughn DJ, et al., Pembrolizumab as Second-Line Therapy for Advanced Urothelial Carcinoma, N Engl J Med, 2017;376:1015-26.

55. Hoffman-Censits J, Grivas P, Van Der Heijden M, et al., IMvigo 210, a phase II trial of atezolizumab (MPDL3280A) in platinum treated locally advanced or metastatic urothelial carcinoma (mUC), I Clin Oncol, 2016;34(suppl 2S; abstr 355).

56. Sharma P, Retz M, Siefker-Radtke A, et al., Nivolumab in metastatic urothelial carcinoma after platinum therapy (CheckMate 275): a multicentre, single-arm, phase 2 trial, Lancet Oncol, 2017:18:312-22.

57. Kiselyov A, Bunimovich-Mendrazitsky S, Startsev V, Key signaling pathways in the muscle-invasive bladder carcinoma: Clinical markers for disease modeling and optimized treatment Int J Cancer, 2016;138:2562-9.

58. Zhou TC, sankin A, Porcelli S, et al, A review of the PD-1/PD-L1 checkpoint in bladder cancer. From mediator of immune escape to target for treatment, Urol Oncol, 2017:35:14-20

9. Zagouri F, Peroukidis S, Tzannis K, et al., Current clinical practice guidelines on chemotherapy and radiotherapy for the treatment of non-metastatic muscle-invasive urothelial cancer: a systematic review and critical evaluation by the Hellenic Genito-Urinary Cancer Group (HGUCG), Crit Rev Oncol Hematol, 2015;93:36-49. 